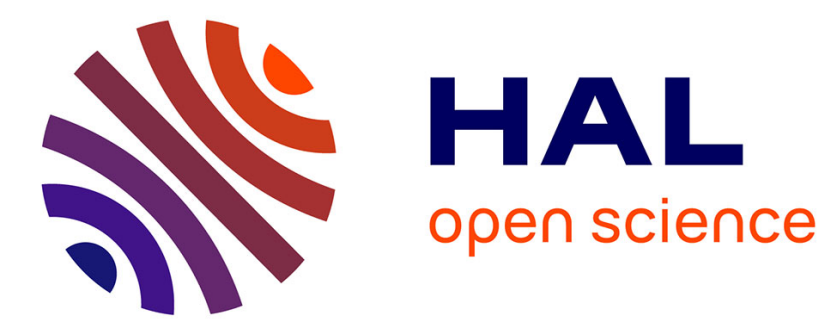

\title{
Prix et coûts de production en agriculture
}

Jean-Marc Boussard

\section{To cite this version:}

Jean-Marc Boussard. Prix et coûts de production en agriculture. 1990, 199, pp.2-5. 10.3406/ecoru.1990.4119 . hal-02703144

HAL Id: hal-02703144

\section{https://hal.inrae.fr/hal-02703144}

Submitted on 1 Jun 2020

HAL is a multi-disciplinary open access archive for the deposit and dissemination of scientific research documents, whether they are published or not. The documents may come from teaching and research institutions in France or abroad, or from public or private research centers.
L'archive ouverte pluridisciplinaire HAL, est destinée au dépôt et à la diffusion de documents scientifiques de niveau recherche, publiés ou non, émanant des établissements d'enseignement et de recherche français ou étrangers, des laboratoires publics ou privés.

\section{(이) $\$$}

Distributed under a Creative Commons Attribution - NonCommercial - NoDerivatives| 4.0 


\section{Jean-Marc Boussard}

\section{Prix et coûts de production en agriculture}

In: Économie rurale. N¹99, 1990. pp. 2-5.

\section{Résumé}

En agriculture, du fait des particularités de ce secteur, les rendements sont pratiquement constants, et l'entrepreneur ne dispose pratiquement d'aucun pouvoir de monopole. La théorie économique classique montre que, dans de telles conditions, le prix doit être égal au coût de production, les seuls écarts possibles venant, soit de l'existence de primes de risque, soit du fait que les prix attribués aux facteurs fixes sont différents de leur coût d'opportunité.

\section{Abstract}

Because of the specificities of agriculture, agricultural production functions are basically homogenous and of degree 1, i.e., with constant returns to scale. Under such conditions, the neoclassical economic theory shows that, under competition, and in the absence of monopoly power, prices should equate production costs. The only possible departures from this arise because of risk (in which case, profits are derived from riskpremia) or because fixed factors are not valued at their opportunity cost.

Citer ce document / Cite this document :

Boussard Jean-Marc. Prix et coûts de production en agriculture. In: Économie rurale. №199, 1990. pp. 2-5.

http://www.persee.fr/web/revues/home/prescript/article/ecoru_0013-0559_1990_num_199_1_4119 


\title{
PRIX ET COÛTS DE PRODUCTION EN AGRICULTURE
}

\author{
Jean-Marc BOUSSARD*
}

\begin{abstract}
Résumé :
En agriculture, du fait des particularités de ce secteur, les rendements sont pratiquement constants, et l'entrepreneur ne dispose pratiquement d'aucun pouvoir de monopole. La théorie économique classique montre que, dans de telles conditions, le prix doit être égal au coût de production, les seuls écarts possibles venant, soit de l'existence de primes de risque, soit du fait que les prix attribués aux facteurs fixes sont différents de leur coût d'opportunité.
\end{abstract}

\section{PRICES AND PRODUCTION COSTS IN AGRICULTURE}

\section{Summary :}

Because of the specificities of agriculture, agricultural production functions are basically homogenous and of degree 1, i.e., with constant returns to scale. Under such conditions, the neoclassical economic theory shows that, under competition, and in the absence of monopoly power, prices should equate production costs. The only possible departures from this arise because of risk (in which case, profits are derived from risk premia) or because fixed factors are not valued at their opportunity cost.

Le calcul des coûts de production est à la mode en agriculture. L'idée que le commerce international devrait être basé sur les avantages comparatifs, et que ceux-ci se traduisent par des différences de coûts, est profondément ancrée dans l'esprit du public. Personne, cependant, ne semble s'interroger sur la notion même de coût de production. Or cette notion est très loin d'être intuitivement évidente, et mérite qu'on s'y arrête. Tel est l'objet de la présente note, dans laquelle je me propose de développer l'idée que le coût de production, en agriculture, doit être normalement très voisin du prix du produit, et que la différence est due, pour l'essentiel, à des phénomènes aléatoires imprévisibles. Comme la chose ne va pas de soi, je devrai d'abord la justifier. J'essaierai d'en indiquer ensuite les conséquences.

\section{COÛTS ET FONCTION DE PRODUCTION}

L'égalité du prix et du coût de production procède en réalité du simple bon sens : l'agriculteur qui vend ses récoltes reçoit en contrepartie une certaine somme d'argent, égale au produit de la quantité par le prix. Cette somme lui sert à payer les facteurs de production variables qu'il a achetés, à payer des redevances pour les facteurs de production fixes qui ne lui appartiennent pas (intérêts d'emprunts, fermages, etc.), et à rémunérer les facteurs de production fixes qui lui appartiennent (son travail et celui de sa famille, son capital propre, etc.). Comme chaque franc qui entre dans l'exploitation est dépensé d'une manière ou d'une autre, il faut bien que la somme des coûts unitaires soit égale aux prix (1).

Ce qui choque, dans une telle interprétation - et qui justifie l'approche traditionnelle de calcul des coûts c'est qu'elle suppose une grande variabilité des prix des facteurs propres (nous donnerons ce nom aux facteurs fixes qui appartiennent en propre à l'agriculteur). Ceci est de nature à troubler l'esprit de ceux qui considèrent les prix comme des constantes de la vie économique, donnés, sinon une fois pour toutes, du moins à intervalles assez espacés pour qu'il soit possible de leur attribuer un minimum de permanence. Par ailleurs, beaucoup de gens n'imaginent pas comment de tels prix peuvent être calculés, ni à quoi ils peuvent servir. Pour le comprendre, il faut avoir recours à la notion de fonction de production, et à quelques notions annexes, au demeurant classiques en théorie de la production.

Une fonction de production est une relation entre les quantités physiques de facteurs de production employés, et les produits qu'ils permettent d'obtenir. C'est une notion tout à fait intuitive, du reste employée sans le mot par nombre d'agronomes : une fonction de réponse à la 
fertilisation, comme en font toutes les stations expérimentales du monde pour présenter leurs résultats, est une fonction de production, au moins partielle. On la note habituellement $f(X, Y)=O$, où $Y$ est un vecteur de quantités produites, et $X$, un vecteur (pas nécessairement de même dimension) d'intrants (2). Y est une quantité en quelque sorte optimale de produit, en ce sens que l'on suppose qu'il est impossible d'augmenter l'un des éléments de Y sans en diminuer un autre, pour $\mathrm{X}$ donné. Inversement, $\mathrm{X}$ est "optimal", en ce sens que, pour Y donné, on ne peut pas diminuer l'un des éléments de X sans en accroître un autre. Cela ne veut pas dire que tous les éléments réalisables de $\mathrm{Y}$ seront effectivement produits pour un $\mathrm{X}$ donné : seuls seront produits ceux qui sont "rentables", les autres restant à l'état virtuel. En ce sens, et selon l'expression de Joan Robinson, la fonction de production est un recueil de techniques possibles, un "book of blue prints", dont seulement une toute petite partie sera effectivement mise en œuvre (3).

Cependant, - c'est là l'intérêt de la notion de fonction de production pour notre sujet - c'est un instrument essentiel pour le calcul des coûts. En effet, dès lors que l'on attribue au producteur un minimum de rationalité sous la forme d'un comportement d'optimisation (en l'absence d'un tel comportement, spontané ou imposé par la concurrence, la notion de coût au niveau d'une collectivité d'exploitations n'a plus guère de sens), et pourvu que la fonction de production n'aie pas une forme mathématique par trop bizarre (il faut que l'optimum existe !), elle permet d'associer un plan de production optimal à un système de prix, et à une dotation en facteurs fixes. En même temps, et sous des hypothèses elles aussi assez générales, elle permet d'attribuer un coût d'opportunité (on parle encore de prix de référence, ou de "shadow price", ou de prix dual) aux facteurs fixes. Ainsi, elle associe une "valeur" aux disponibilités en facteurs fixes, et permet donc un calcul de coûts de production qui échappe complètement aux "conventions comptables" que l'on critique à bon droit si souvent. Le calcul est en quelque sorte complètement interne à la situation objective de l'exploitation. Cela ne veut pas dire qu'il n'y ait pas du tout de conventions à faire pour effectivement réaliser le calcul. Trois sont spécialement importantes :

$1^{\circ}$ ) Il faut d'abord savoir quoi maximiser. De ce point de vue, il est tout aussi logique qu'arbitraire de maximiser quelque chose qui ressemble à une marge brute :

$$
F=y^{\prime} Y-x_{1}^{\prime} X_{1}
$$

où $X_{1}$ représente les facteurs variables. Cependant, une telle fonction d'objectif est tout de même bien plus générale que celle qui est prise implicitement par les gens qui effectuent les calculs comptables classiques, et qui maximisent le profit, en attribuant des prix plus ou moins arbitraires aux facteurs fixes. Il n'en demeure pas moins qu'elle est susceptible d'une grave objection, celle de l'hypothèse implicite d" "avenir certain" qui la sous-

2. Dans ce qui suit, nous présenterons les quantités par des lettres majuscules, et les prix correspondants par des lettres minuscules.

3. Il va de soi aussi que cette définition, assez abstraite, de la fonction de production n' a que peu de chose à voir avec des instruments comme la "fonction Cobb Douglas" ou la "fonction Translog" que trop d'économètres amateurs ont tendance à essayer de faire passer comme la seule expression empirique possible de la notion.

4. En effet, dans la formule précédente, les prix sont supposés connus, et donnés de façon exogène. tend (4). La discussion de ce point romprait le fil du raisonnement, et nous la reporterons plus bas (5).

$2^{\circ}$ ) Il faut savoir quels sont les facteurs fixes et variables. Ici, il est raisonnable de se laisser à nouveau guider par la théorie économique : un facteur de production n'est pas fixe ou variable par nature. Un facteur fixe est celui dont le coût d'opportunité se situe entre le prix auquel il est possible de s'en débarrasser (c'est la valeur de liquidation, éventuellement négative, s'il faut par exemple payer un casseur pour venir chercher une épave de voiture...) et le prix auquel on peut l'acheter. Ainsi a-t-on les moyens (au moins théoriquement) (6) de définir de façon objective ce qui est fixe et variable. En même temps, on se fixe des bornes supérieures et inférieures pour l'intervalle de variation du coût d'opportunité, et on se délivre de l'angoissante question que se posent toujours les comptables sur la significativité du prix du marché d'un facteur sur lequel la vaste majorité des entreprises n'effectue aucune transaction.

$3^{\circ}$ ) Enfin, et surtout, il faut se donner une forme analytique pour la fonction de production. Tout choix dans ce domaine sera discutable et discuté. La nomenclature même des produits va poser un problème : le coût d'opportunité du travail n'est pas le même en juillet et en décembre. Faut-il donc considérer ces deux types de travaux comme deux produits différents ?

Il est vrai que la réponse à des questions de ce type est essentielle à qui veut réellement obtenir la solution du problème d'optimisation évoqué plus haut. Mais pour ce qui nous concerne ici, ce n'est sans doute pas nécessaire. Il suffit d'admettre une propriété tout à fait générale de la fonction de production pour établir l'égalité du prix et du coût de production. Un vieux théorème, dû à Euler, permet en effet d'établir cette proposition dans le cas des fonctions de production homogènes et de degré 1 , lorsqu'on maximise le profit, et que les facteurs sont rémunérés à leur coût d'opportunité. Or quelle est la signification du fait que la fonction de production est homogène et de degré 1 ? C'est l'existence de "rendements constants", le fait que, s'il est possible de cultiver du blé sur 1 ha avec certains moyens de production, on puisse en faire autant sur deux ha avec deux fois plus de moyens (7). Les fonctions de production agricoles sont-elles dotées de telles propriétés? On en discute, et la discussion durera sans doute longtemps, tant il est vrai qu'il est impossible de prouver une proposition aussi générale. Ce que l'on peut dire, c'est que le contraire n'a jamais été formellement démontré, en dépit des nombreux efforts effectués dans ce sens (8). Dans ces conditions, il est tout aussi facile d'admettre l'homogénéité de degré 1 de la fonction de production agricole, ce qui entraîne, dans le cadre d'hypothèses qui vient d'être défini, l'égalité des coûts de production avec les prix, dès lors que l'on évalue les facteurs fixes à leur coût d'opportunité.

5. Remarquons, du reste, que le calcul comptable traditionnel suppose lui aussi l'avenir certain, et pour les mêmes raisons.

6. Pratiquement, le calcul peut être effectué à partir d'un algorithme du type "branch and bound", comme ceux que l'on utilise dans la programmation linéaire en nombres entiers.

7. La fonction de production $f(X, Y)$ est homogène et de degré 1 si pour tout réel $\lambda$ positif : $f(X, Y)=O$ entraine $f(\lambda X, \lambda Y)=O$. On trouvera des détails dans tous les ouvrages de base sur la théorie de la production, éventuellement chez Boussard (1987, p. 87.104).

8. Beaucoup de gens ont cru avoir trouvé des preuves empiriques du contraire. Aucune $n$ 'est indiscutable. 


\section{LIMITES ET CONSÉQUENCES DE L'ÉGALITÉ DES PRIX ET DES COÛTS DE PRODUCTION}

Nous espérons ainsi avoir montré le caractère raisonnable de l'assertion selon laquelle le prix est égal au coût de production en agriculture. En même temps, nous avons montré à quelles conditions cela était vrai, et ce que cela impliquait pour l'évaluation des facteurs fixes. Il reste à en tirer les conséquences. Auparavant, il nous faut lever l'hypothèque de l'hypothèse d'“avenir certain', évoquée plus haut.

\section{A) Profit et prime de risque}

De fait, si l'on tient compte de l'aversion des producteurs pour le risque, ainsi que du caractère fondamentalement aléatoire aussi bien des fonctions de production que des prix, l'analyse précédente doit être révisée. Ou bien il faut maximiser autre chose que la formule (1), par exemple, l'espérance d'utilité du profit, comme le recommande la théorie officielle en la matière, ou bien il faut maximiser l'espérance de profit (ou encore le profit anticipé, ce qui peut ĉtrc différent), mais sous une contrainte particulière, exprimant que le risque pris dans une solution donnée du problème de maximisation n'est pas excessif. Dans les deux cas, on montre facilement qu'il existe alors une différence plus ou moins importante entre l'espérance de recette, $y$ ' $Y$, et l'espérance de coût, $X$ ' $X$. Cette différence peut être assimilée à un profit. Il s'agit en réalité de quelque chose d'entièrement différent, qui devrait plutôt s'appeler prime de risque, et qu'il est de toute manière hors de question de maximiser (9). En ce sens, et en ce sens seulement, il peut exister de fortes divergences entre le prix et le coût de production, divergences d'autant plus importantes que la culture considérée est plus risquée. Encore cette différence disparaît-elle si l'on admet que l'important (du moins pour le choix des productions) n'est pas le prix réel des produits agricoles, mais leur "équivalent certain", qui, lui, reste égal au coût de production. Une autre façon de traiter le problème est de considérer le risque comme une sorte de facteur de production supplémentaire (ou plutôt, comme un "antifacteur"), et de lui attribuer sa part dans le calcul des marges brutes. C'est même là, sans doute, la meilleure méthode, et la plus cohérente, de traiter le problème.

A côté de cela, le caractère aléatoire de la production fait que, pour une année donnée, il existe évidemment toujours une différence entre l'équivalent certain du prix observé, et le coût de production planifiè par l'agriculteur. Naturellement, si les anticipations de l'agriculteur sont correctes en moyenne, cette différence, sur un grand nombre d'années et un grand nombre d'agriculteurs, doit avoir une espérance mathématique qui converge vers zéro. Il ne vaut donc pas la peine de s'y apesantir ici, sauf peutêtre pour noter à quel point ces perturbations par rapport à des variables de surcroît inobservables (les anticipations) sont de nature à compliquer l'inférence statistique quand on l'applique à l'économétrie du secteur agricole.

9. La maximisation de ce profit reviendrait à sélectionner dans le plan de production les cultures les plus risquees, pour lesquelles la prime de risque est ćvidemment la plus importantc. C'cst du reste ce que font, hélas, bien trop de conseillers de gestion, prisonniers qu'ils sont de schémas comptables sans rapport avec la realite economique.

10. Heureusement, de telles énormités n'ont pas cours en France. Mais c'est sur la base de calcul analogue que l'on voit certains universitaires Américains (ou mème Français) conseiller aux Africains l'abandon des cultures céréalières.
Il peut enfin arriver que les anticipations ne soient jamais correctes. Il faut avouer que la théorie économique ne nous est plus d'un grand secours dans une telle situation.

\section{B) Faible intérêt des calculs de coûts de production}

La première leçon à tirer de l'analyse précédente est triviale : si elle est correcte, il est tout à fait inutile de s'acharner sur les calculs de coûts de production, puisque ceuxci sont à peu de chose près égaux aux prix, à la prime de risque près.

En pratiquant l'analyse comptable traditionnelle, on pourra, au mieux, mettre en évidence des différences de primes de risque. Cela ne servira pas à grand chose en matière de politique agricole. Au pire, on en tirera des conclusions absolument erronées : par exemple, en utilisant le même prix pour évaluer le travail dans une "grande", et une "petite" exploitation, on n'aura pas de peine à faire apparaître le "désavantage comparatif"' de la petite dans toutes les productions intensives en travail, puisque, dans une telle situation, on aura systématiquement gonflé le prix réel du travail chez le petit agriculteur. Ainsi conseillerat-on au céréalier Beauceron de s'établir producteur de lait (10).

La vérité, c'est que les facteurs fixes de chaque agriculteur sont rémunérés à un prix différent de ceux des autres. Cela entraîne que des techniques de production extrêmement différentes peuvent coexister, et rester compétitives, en dépit de la recherche du profit maximum par les producteurs : ainsi, la production de riz pluvial, en Afrique ou en Asie du Sud-Est, se fait-elle pratiquement sans capital (du moins, sans aucune machine), tandis que, au Texas, le même produit est obtenu à partir de techniques qui ne réclament qu'un nombre infime d'heures de travail par quintal. Pourtant, les deux modes de production sont parfaitement compétitifs, et se font concurrence sur des marchés pratiquement libres.

La chose importante à considérer, dans les calculs de coûts de production, ce n'est donc pas le coût lui-même, mais bien plutôt la rémunération qu'une technique donnée est susceptible de garantir à tel ou tel facteur de production. Si une technique de production, ou un produit, est susceptible de garantir à l'ensemble des facteurs habituellement fixes dans un pays donné, une rẽmunération qui tombe dans la fourchette de définition de ces facteurs fixes, alors il y a beaucoup de chances pour que quelques agriculteurs, au moins, de ce pays, soient dans la position de l'adopter (11). Dans cet ordre d'idée, il est important d'observer que la réponse à cette question dépend du prix et des "coefficients techniques" de la culture considérée, mais, tout autant, du prix et des coefficients techniques des cultures et des techniques susceptibles d'entrer en compétition avec elle : ainsi, un changement du prix du lait est-il susceptible de modifier la compétitivité du

11. En pratique, la preuve d'une telle situation est en général assez difficile à faire. II est probablement plus simple, et absolument équivalent, lorsqu'on a des doutes sur un sujet de ce type, de le lever à l'aide d'un modèle de décision des agriculteurs, du type "programme linéaire". Aussi bien, les données à recueillir, tant pour la mesure des coûts de production, que pour la programmation linéaire, sont-elles les mêmes. Toutefois, l'approche "programme linéaire"', présente l'intérêt de mettre en évidence le caractère global de toute étude d'avantages comparatifs, en particulier l'influence du prix d'une culture sur la rentabilité d'une autre. 
blé dans un pays donné. Voilà un résultat simple de théorie de la production qui n'est pas pris du tout en considération par la technique des PSE (12).

\section{C) La dynamique des coûts}

A noter que, normalement, la différence entre prix du marché et coût d'opportunité devrait être au détriment des agriculteurs, puisque, par hypothèse, les facteurs fixcs lc sont parce que leur productivité marginale est plus faible que leur prix d'acquisition. Mais il en était différemment au moment de leur acquisition (autrement, ils n'auraient pas été achetés). En réalité, au moment de l'achat, il est permis de penser que la productivité marginale était juste égale au coût. Il importe de noter, du reste, de ce point de vue, que l'endettement accroissant les risques de faillite, la prime de risque augmente très vite avec l'endettement, contribuant ainsi à abaisser rapidement la productivité marginale des facteurs de production achetés à crédit par rapport à celle des mêmes facteurs financés sur fonds propres.

Par la suite, l'évolution de la productivité marginale de chaque actif est gouvernée par deux phénomènes : d'une part, le désendettement lié au remboursement des emprunts (l'enrichissement de l'agriculteur vers la fin de son cycle de vie) tend à diminuer la prime de risque, et donc à augmenter la productivité marginale des actifs. De fait, on voit beaucoup d'exploitations grandir quelques années après leur installation parce que des facteurs, fixes au tout début de l'activité de l'agriculteur, deviennent maintenant variables ; d'autre part, les conditions socioéconomiques n'étant jamais constantes, le régime des prix change, changeant par là même la productivité marginale de chaque catégorie d'actif. En général, ces changements, de même que le progrès technique, tendent à diminuer la productivité marginale des actifs existants, qui par là deviennent fixes. Eventuellement, la productivité marginale (ou la "valeur d'usage", comme on voudra) de chaque actif devient inférieure aux intérêts des dettes contractées, et l" "agriculteur en difficulté" voit son revenu amputé d'une fraction de ses remboursements d'emprunts.

Ainsi, le temps fait varier les rémunérations. En fait, au cours du temps, les rémunérations admissibles pour les facteurs de production changent. Lorsqu'un pays se développe, il arrive un moment où la rémunération du travail fournie par les techniques de production en vigueur jusque là devient inférieure, même pour les plus pauvres, à ce qu'ils peuvent obtenir d'autres sources, fut-ce l'assistance au chômage (ou même la vie sur les déchets des riches, comme cela se passe dans de nombreux bidonvilles du Tiers-Monde). Alors, ces techniques disparaissent, et les cultures correspondantes sont abandonnées, à moins qu'il ne soit possible de mettre en œuvre d'autres techniques, moins intensives en travail et plus intensives en capital que précédemment. Ici encore, la notion comptable de coût de production n'aide pas beaucoup à comprendre ce phénomène, pourtant central dans l'appréciation de l'évolution de l'agriculture.

La discussion précédente entraîne une autre conséquence : il y a longtemps que les économistes se sont interrogés sur la causalité du haut (ou faible) prix des terres. Déjà, au début du $19^{\mathrm{e}}$ siècle, Ricardo se posait cette question : "Le blé ne renchérit pas parce qu'on paie une rente. C'est au contraire parce que le blé est cher que l'on paie une rente'. (Ricardo, 1821 ; cité par Flichmann et Jacquet, 1988). I.'idée est en effet que l'accroissement du prix du blé, en permettant de payer un prix élevé pour la terre, est responsable de son prix élevé.

Il en est de même pour tous les facteurs fixes, et ce point de vue donne une nouvelle dimension aux analyses de politique agricole.

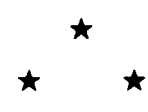

Ainsi, la théorie de la production conduit-elle à traiter les coûts de production d'une façon tout à fait différente de la comptabilité classique, qui cherche à faire apparaître un profit. En fait, il est permis de penser que c'est une grave erreur d'adopter, par mimétisme, pour calculer les coûts de l'exploitation agricole, des techniques de calcul valables pour apprécier la répartition des bénéfices dans les sociétés anonymes. Dire cela n'implique pas que l'on adhère à certaines théories à la mode sur le fondamentalisme agraire. Nous sommes restés ici dans le cadre strict de l'analyse de type "néoclassique" (13). Mais nous avons essayé de repérer les spécificités de l'agriculture, et d'en tenir compte. C'est bien là, après tout, le rôle d'un économiste de l'agriculture.

\section{RÉFÉRENCES BIBLIOGRAPHIQUES}

FLICHMANN G. et F. JACQUET (1988). - Intensification et efficacité en agriculture. Economie Rurale (183), janvier-février.

\author{
BOUSSARD J.M. (1987). - Economie de l'Agriculture, Economica, \\ Paris.
}

12. Producer Subsidy Equivalent : une technique de calcul supposée permettre l'évaluation des subventions reçues par les agriculteurs d'un pays ; déduits du prix payé aux producteurs, ces PSE sont supposés permettre le calcul d'un prix de référence net de subvention, qui, lui-même, servirait de base à l'éva. luation de l'avantage comparatif d'un pays dans une production.
13. Il n'y a pas bien sür "une" théorie néoclassique, mais une foule de modèles différents, éventuellement contradictoires; en fait, presqu'autant que d'èconomistes. 